\title{
CAPILLARY OPTICAL GUIDING EXPERIMENTS FOR THE NRL LASER WAKEFIELD ACCELERATOR*
}

\author{
T. G. Jones, C. I. Moore, A. Ting, P. Sprangle, \\ Plasma Physics Division, Naval Research Laboratory, Washington, DC \\ D. Kaganovich, LET Corp., Washington, DC \\ K. Krushelnick, Imperial College, London, United Kingdom
}

\begin{abstract}
Extended propagation of terawatt (TW) laser pulses has been shown in plasma channels formed with a capillary discharge[1]. Capillary optical guiding experiments at NRL utilize plasma channels formed using both laser ablation and electrical discharge. Present experiments aim to develop laser preionization and ablation from capillary walls as a formation technique for the guiding plasma. Electron density measurements of laser-formed plasmas within the capillary yield values as low as $10^{18} \mathrm{~cm}^{-3}$. These densities hold promise for optimization of plasma conditions for standard laser wakefield acceleration (LWFA). Experiments also aim to characterize plasma ablation dynamics and plasma channel conditions within the capillary using a variety of imaging and other diagnostics. Imaging diagnostics include axial shadowgraphy. Capillary guiding diagnostics such as transmission efficiency, output beam mode imaging, timeresolved backward-scattered Raman spectra, forward Raman spectra, and Thomson scattering imaging are also employed.
\end{abstract}

\section{INTRODUCTION}

Advances in laser technology have enabled experiments using laser pulses focused to extremely high intensity ( $\mathrm{I} \geq$ $10^{19} \mathrm{~W} / \mathrm{cm}^{2}$ ). Such intensities have opened the exploration of new parameter regimes in both atomic and plasma physics, as well as potentially important applications of very high intensity light, such as advanced particle acceleration techniques [2].

However, the volume over which such high fields exist is not large, since it is usually necessary to focus the beam to a very small spot size, resulting in short diffraction distances. This diffraction length (Rayleigh range) is given by $\pi r_{o}^{2} / \lambda$; where $r_{o}$ is the $1 / \mathrm{e}^{2}$ radius of the beam waist and $\lambda$ is the laser wavelength. For example, if a laser operating at a wavelength of $1 \mu \mathrm{m}$ is focused to a 20 $\mu \mathrm{m}$ diameter spot, the diffraction distance is only $0.3 \mathrm{~mm}$. Thus, proposed applications for high intensity lasers will only become practical if the length over which the beam maintains its focused intensity can be extended significantly. A means of channeling the beam over many Rayleigh lengths is necessary.

In an underdense plasma, channeling of laser pulses is possible if an appropriate radial variation in the index of refraction, $\eta$, can be produced across the laser profile. The index of refraction in plasma, for radiation of frequency $\omega$, is given by

$$
\eta^{2}=1-\frac{\omega_{p e}^{2}}{\omega^{2}}
$$

where $\omega_{p e}=\left(\frac{4 \pi n_{e} e^{2}}{m_{e}}\right)^{1 / 2}$ is the electron plasma frequency, $n_{e}$ is the plasma electron density, and $m_{e}$ is the mass of the plasma electron. For a cylindrical volume of plasma, a refractive index which is centrally peaked radially can produce focusing or "channeling", similar to the effect produced by an optical fiber.

Preformed plasma channels capable of guiding TW laser pulses have been formed with a capillary discharge[1]. However, these discharge-formed channels have been measured to have densities $\sim 10^{19} \mathrm{~cm}^{-3}$ or higher [3]. Standard LWFA requires densities for which the plasma period, $\tau_{p}=2 \pi / \omega_{p e} \sim \tau_{L}$, where $\tau_{L}$ is the laser pulse length. For a pulse length of $400 \mathrm{fs}$, the matching density is $\sim 10^{17} \mathrm{~cm}^{-3}$. Furthermore, discharge-formed capillary plasma channels are maintained by plasma currents of several hundred amps, generating magnetic fields on the order of $1 \mathrm{~T}$. Such magnetic fields would likely increase the emittance of an accelerated electron beam. Laser ablated capillary plasmas are free of discharge-generated magnetic fields, and as discussed in Sec. 3, have been measured to have $n_{e} \sim 10^{18} \mathrm{~cm}^{-3}$. These characteristics are promising for application to guiding for laser acceleration in general and for standard LWFA in particular.

The present experiments aim to explore the dynamics of laser preionization and ablation from capillary walls, and to develop this technique as a means of forming a plasma channel for guiding a TW laser pulse.

\section{EXPERIMENTAL SET-UP}

Capillaries composed of plastics[1], metals, and glass[4] have been used for high power optical guiding experiments at NRL. The present ablation imaging experiments utilize polyethylene capillaries with 500 micron diameter x $12 \mathrm{~mm}$ length holes. Experiments are

\footnotetext{
* This work is supported by DOE and ONR.
} 


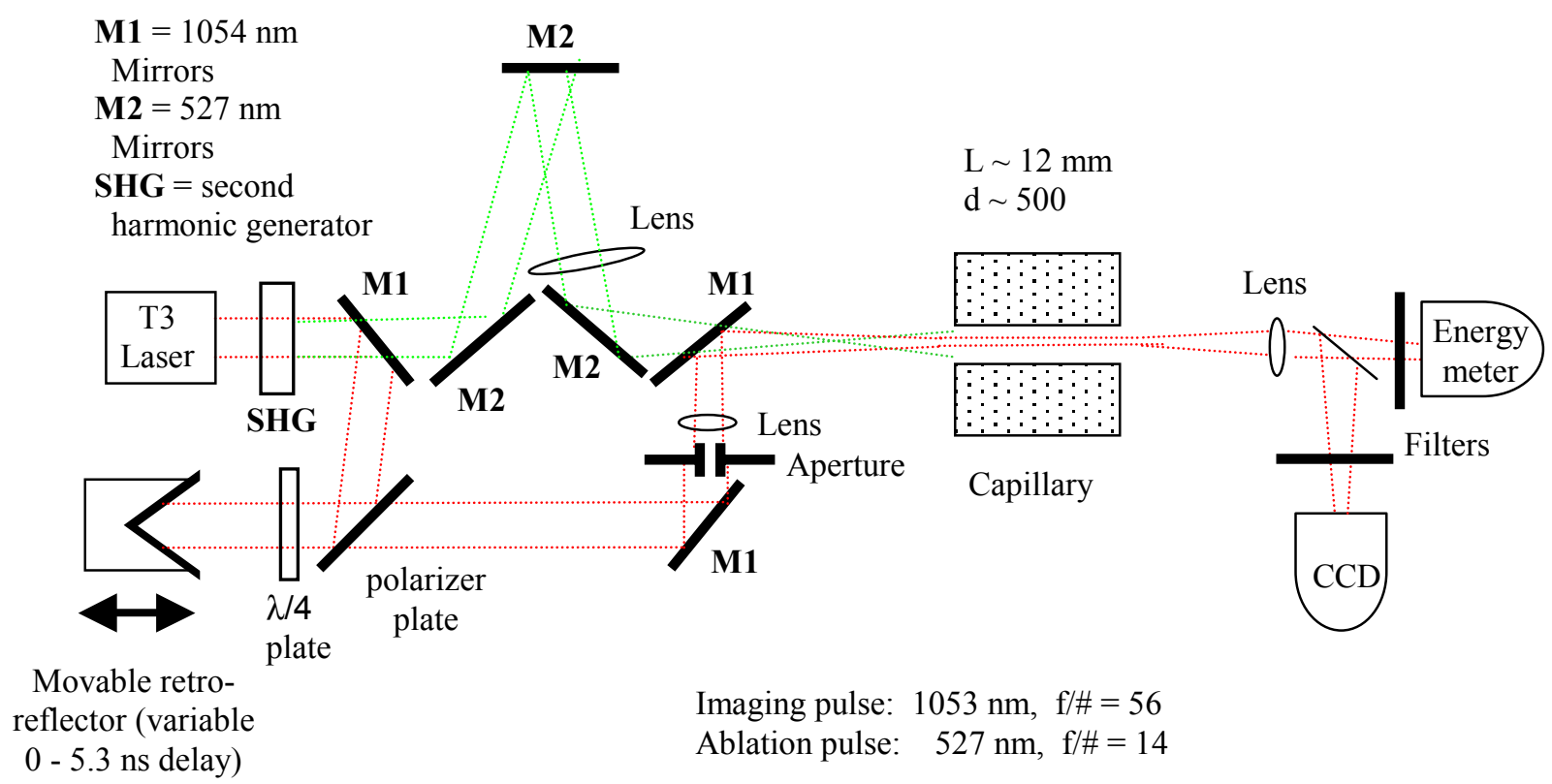

Figure 1: Schematic diagram of the setup for shadowgraphic imaging of a subpicosecond laser-ablated capillary channel.

performed in a vacuum chamber, which is kept at pressures below $10^{-4}$ Torr.

Laser acceleration and TW optical guiding experiments at NRL utilize the T-cubed laser system. This laser is a chirped pulse amplification system operating at $1054 \mathrm{~nm}$, with 400 fs compressed pulse duration, and beam diameter of $43 \mathrm{~mm}$. Laser pulses travel through a $\mathrm{KD} * \mathrm{P}$ second harmonic generator (SHG), which can convert up to $50 \%$ of the light into $527 \mathrm{~nm}$. The fundamental and second harmonic components of the pulse are then separated, with the $527 \mathrm{~nm}$ pulse used for ablation from the capillary walls (due to a slightly higher ionization efficiency), and the $1054 \mathrm{~nm}$ pulse is used as a diagnostic probe of the plasma. A schematic diagram of the capillary ablation experiment is shown in Figure 1.

The delay between the ablation pulse and the probe pulse is adjustable via a delay stage for the probe pulse utilizing a thin film polarizer, a quarter-wave plate, and a movable retroreflector. This delay can be adjusted from 0 to $5.3 \mathrm{~ns}$. The $1054 \mathrm{~nm}$ probe pulse is then available as a guided probe pulse at high intensities, and as an imaging beam at lower intensities.

The focusing geometry and alignment of the ablating laser pulse with the capillary is critical to the uniformity of the plasma channel. Assuming an ablated plasma density proportional to the local laser fluence on the capillary surface, an ablation beam with large $f / \#$ is desirable for axial plasma uniformity. However, an upper limit on the $f / \#$ is determined by the maximum ablation pulse energy and the minimum ablation fluence required to produce a plasma. In addition, a large $f / \#$ requires more precise alignment of the ablation pulse with the capillary axis to avoid azimuthal asymmetry of laser fluence on the wall. The ablation pulse in our experiment was focused with $f / \# \sim 16$, yielding an axial variation in fluence on the capillary wall of a factor of 6 and intensities of few $\times 10^{12}$ $\mathrm{W} / \mathrm{cm}^{2}$. The ablation pulse was focused $7 \mathrm{~mm}$ in front of the capillary entrance, producing a diverging beam which nearly covered the inner surface of the capillary.

Plasma ablation dynamics, including azimuthal symmetry, are diagnosed with axial shadowgraphy using the probe pulse. Shadowgraphy utilizes a collimated or nearly collimated beam to produce a projection of axially integrated information about the plasma profile at the capillary exit plane. At low plasma densities where $\omega_{p e}$ $<<\omega$, the probe beam is refracted by a density gradient so that it is deflected in the direction of lower density. At higher plasma densities the beam is reflected from the plasma boundary where $\omega_{p e}=\omega$. To generalize, for low densities this diagnostic technique produces a brightening of the probe beam image at locations of localized minima in the axially integrated density. The probe beam was imaged by a lens system with $f / \# \sim 2$ onto a CCD camera.

Additional diagnostics include energy monitors measuring input and output energies of high power probe pulses to determine optical energy transmission efficiency. The transverse beam mode structure can be imaged for determination of optical guiding. Also, backscattered light generated by stimulated Raman scattering (SRS), for which $\omega_{S R S}=\omega-\omega_{p e}$, can be recorded by a spectrometer for high power probe pulses. The shift of the SRS 
spectral peak yields the plasma density within the capillary.

\section{EXPERIMENTAL RESULTS}

Preliminary experiments with laser ablation did not produce optical guiding, as indicated by energy transmission measurements and probe beam mode imaging at the capillary exit. However, plasmas of sufficient density were produced so that by $10 \mathrm{~ns}$ after the ablation pulse, transmission of the probe pulse had decreased to zero.

A typical sequence of shadowgraphs during plasma ablation is shown in Fig. 2. The ablation pulse had approximately $100 \mathrm{~mJ}$ of energy. Each frame is a different shot, with the the ablation pulse at $t=0$, and the probe pulse at the indicated time. Shadowgraph structure was reproducible for a given ablation pulse energy, probe pulse delay, and alignment with the capillary axis. The six frames shown in Fig. 2 are a sample from a set of shots with $0.5 \mathrm{~ns}$ delay increments between 0.5 and 5.3 $\mathrm{ns}$, and the frame at $\mathrm{t}=0$ taken without an ablation pulse.
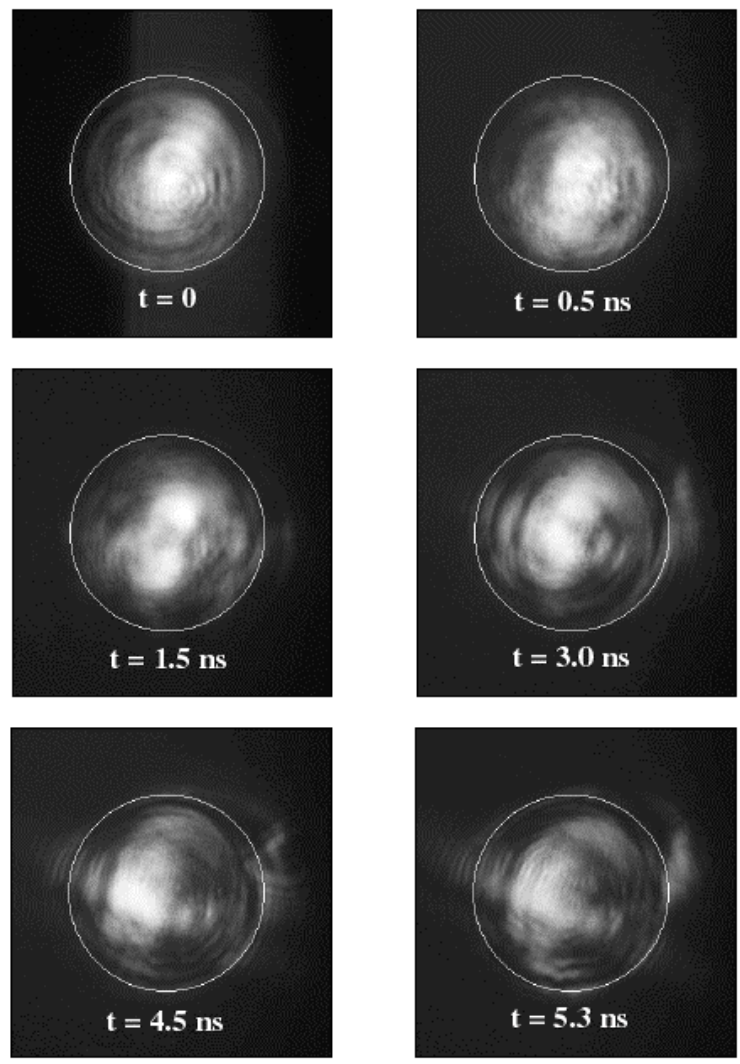

Figure 2: A sequence of shadowgraphs shows plasma ablation from capillary walls during the first few nanoseconds after the ablation pulse. An outline of the 500 micron capillary exit is drawn as a guide. A nonlinear contrast map was used to enhance details of the beam edges.

Shadowgraphs during the first 1.5 ns show some concentration of the probe pulse towards the center of the capillary exit, suggestive of ablated plasma along the capillary walls. At $\mathrm{t} \sim 1.5 \mathrm{~ns}$, the shadowgraph has a double-peaked intensity profile, indicating two local minima of axially integrated density. Refraction of the lower part of the beam at $\mathrm{t}=1.5 \mathrm{~ns}$, indicates plasma has moved into this region, at a velocity $\sim$ few $\times 10^{6} \mathrm{~cm} / \mathrm{s}$.

At late times, $t>4.5 \mathrm{~ns}$, a bulk shift of the probe beam image towards the left can be seen. This is interpreted as roughly uniform refraction of the probe beam by a plasma density gradient, with density increasing from left to right. Such a density gradient might be caused by a horizontal tilt of the ablation pulse resulting in higher fluence on the right side of the capillary wall. Assuming axial uniformity and a uniform transverse density gradient, the change in the refractive index, $\nabla \eta \sim d / 2 L^{2}$, where $d$ is the image displacement, and $L$ is the capillary length. For an image displacement of 150 microns, as seen for $\mathrm{t}>4.5 \mathrm{~ns}$, Eq. 1 yields a density gradient estimate of $\Delta n_{e} \sim 10^{18} \mathrm{~cm}^{-3}$ across the capillary diameter. This estimate of the density gradient is consistent with backscattered SRS spectra showing plasma channel densities of $1-2 \times 10^{18} \mathrm{~cm}^{-3}$.

The ablation beam focal spot was measured subsequent to the experiment, indicating poor beam quality (approximately 4 times diffraction limited) and slight ellipticity. Both of these defects were found to be caused by the SHG. This may partly explain the double-peaked $(\mathrm{m}=2)$ azimuthal asymmetry of the shadowgraph images seen at $\mathrm{t} \sim 1.5 \mathrm{~ns}$. Efforts to improve the ablation pulse beam quality, as well as its alignment with the capillary axis are presently underway.

\section{SUMMARY}

A pump-probe experiment to study laser ablation of capillary plasmas has been performed. Laser ablation was achieved using a subpicosecond diverging laser pulse coaxial with the capillary. Shadowgraphic imaging using a second subpicosecond pulse with adjustable delay yielded axially integrated information on plasma ablation dynamics. Plasma ablation velocities were estimated at a few $x 10^{6} \mathrm{~cm} / \mathrm{s}$. Estimated density gradients inferred from refraction of the probe beam yield $\Delta n_{e} \sim 10^{18} \mathrm{~cm}^{-3}$ across the capillary diameter, and are consistent with independent density measurements using backscattered SRS. These densities are lower than those produced by capillary discharge, and show promise for producing channels suitable for LWFA with the T-cubed laser. Work is continuing on developing laser ablated plasma channels capable of guiding TW laser pulses for LWFA.

\section{REFERENCES}

[1] D. Kaganovich, et al., Phys. Rev. E 59 R4769 (1999).

[2] T. Tajima and J. M. Dawson, Phys. Rev. Lett. 43, 267 (1979); P. Sprangle, E. Esarey, A. Ting, and G. Joyce, Appl. Phys. Lett. 53, 2146 (1988).

[3] D. Kaganovich, et al., Appl. Phys. Lett. 75, 772 (1999).

[4] S. Jackel, R. Burris, J. Grun, A. Ting, C. Manka, K. Evans, and J. Kosakowskii, Optics Letters 20, 1086 (1995). 